\title{
Revenue Management for Low-Cost Providers
}

\author{
Benjamin Marcus, Suffolk University \\ Chris K. Anderson, Cornell University
}

Low-cost providers have emerged as important players in many service industries, the most predominant being low-cost, or the so-called discount airlines. This paper presents models and results leading toward understanding the revenue management outlook for a discount pricing firm. A framework and model is formulated specifically for the airline industry, but is generalizable to low-cost providers in similar revenue management settings. We formulate an optimal pricing control model for a firm that must underprice to capture a segment of exogenous demand. Two specific model formulations are considered: a continuous deterministic version, and a discrete stochastic version. Structural results are derived for the deterministic case, providing insight into the general form of optimal underpricing policies. The stochastic results support the structural insight from the deterministic solution, and illuminate the effect of randomness on the underpricing policies.

\section{Introduction}

Low-cost providers have emerged as important players in many service industries. In particular, low-cost carriers in the airline industry have enjoyed significant success over the past decade or more. These firms typically have simple fare class structures, fly point-to-point service, and use the lowertraffic airports, all of which contribute to their ability to consistently offer low fares. Well known carriers - including JetBlue, Ryanair, and Westjet - are only a few of the growing number of firms that employ similar discount pricing strategies. Beyond airlines, low-cost strategies have also been used successfully in other sectors of the travel and tourism industry, including car rentals, hotels and resorts.

Many firms, including those described above, face the problem of selling inventory by a fixed date, with no ability to reorder and no salvage value for unsold goods. When demand for their capacity is stochastic, and realized through products that compete for the same limited resource, selling optimally is extremely challenging. A simple example illustrates the sales problem. Consider the 
following situation in which an airline's capacity is a single seat. Suppose the firm posts a price of $p_{t_{1}}$ today. A customer arrives, and is willing to pay $p_{t_{1}}+\delta$, known as the reservation price, for the seat. The seat is sold if and only if $p_{t_{1}}+\delta>p_{t_{1}}$. Later in the booking period, another customer arrives and is willing to pay $p_{t_{1}}>p_{t_{1}}+\delta$. If the seat was sold to the first customer, no sale will be made. If the seat is still available, the second customer will make a reservation if the posted price at $t_{2}$ is less than or equal to their reservation price. Now the dilemma is clear. If the firm knew there was no second customer they should post a price of $p_{t_{1}}+\delta$ today, since otherwise $\delta$ value is lost or no sale will be made. And if they knew that there would be a second customer they should post a price above $p_{t_{1}}+\delta$, since the second is willing to pay more.

Unfortunately, the time of arrival and reservation prices of customers are unknown and stochastic, and thus difficult to predict. The uncertainty in demand presents the greatest challenge in revenue management applications, both in practice and theory. Early airline research (Littlewood, 1972 and Belobaba, 1987) utilized marginal rules to determine when airlines should stop selling discounted seats (when they should close the discount fare class). These marginal rules are based on the assumption that customers with lower willingness to pay attempt to book early. Extensions to these rules (Pfeifer, 1989) have been developed to account for customers arriving more randomly. These extensions help account for the diversion of customers across product classes, in essence trying to limit the number of customers paying less than their willingness to pay. As in the early work, much of the airline seat allocation and pricing research (see McGill and van Ryzin, 1999 and Weatherford and Bodily, 1992 for relevant reviews) hinges upon structured fare products to help segment customers in accordance with their willingness to pay. More recently, owing in part to the proliferation of discount carriers, research has started to focus on seat allocation in the absence of fare classes (Hopperstad and Belobaba, 2004 and Tavana, 2004). With the removal of fare classes, it becomes natural to view revenue management as a price-based control problem, the complement to the allocation-based control historically practiced in the airline industry.

Dynamic pricing, the hallmark of price-based revenue management, is the usual demand management mechanism in many practical settings. As a result, there has been considerable research devoted to understanding this approach and its implications. Gallego and van Ryzin (1994) develop an intensity control model for a single product in an environment with stochastic demand. In Gallego and van Ryzin (1997) they extend this work to consider the case of multiple products in a network environment. Feng and Gallego (1995) explore the optimal timing of price changes when only a single change is allowed over the selling season. An extension to this work is provided by Feng and Xiao 
(2000b), in which multiple monotonic price changes are allowed, either markup or markdown, and chosen from a predetermined set. In a related work, Feng and Xiao (2000a) allow for multiple prices changes that are reversible, i.e., not monotonic. Netessine (2006) also examines a case where price changes are costly, resulting in changes being limited in frequency rather than number. You (1999) considers the joint decision of which fare classes to open and how prices should be set dynamically for the available fares. And Zhao and Zheng (2000) and Feng and Gallego (2000) address optimal dynamic pricing under time dependent demand characteristics. For a more detailed background than provided here, a good introduction to dynamic pricing is provided in Talluri and van Ryzin (2004b), and helpful overviews of the academic literature can be found in Elmaghraby and Keskinocak (2003) and Bitran and Caldentey (2003). In the present work, a low-cost firm also sets prices dynamically, with the only restriction being that prices are bounded above by a maximum related to the price set by another service provider. We first consider the case when these changes are made continuously, and next, the case when the changes are at discrete-time intervals.

One of the assumptions found in many of the works cited above is that the demand processes for available fare products are independent and not control dependent. Although this simplifies the analysis of the revenue management setting, it is clearly an unrealistic model of actual consumer behavior, and may create problems in practice (Wilson et al., 2006). Though several of the dynamic pricing models attempt to address this limitation, they only partially do so: "While these models allow demand to depend on price (the control in this case), they assume only one product is sold at one price at any point in time. Thus, customers face a binary choice: to buy or not to buy." Talluri and van Ryzin (2004a) To more fully address the deficiencies of the independent demand assumption, several researchers have recently developed consumer choice revenue management models, in which individual consumer preference and product selection are modeled explicitly. A single leg, multiple-fare class model with general discrete choice customer behavior is considered in Talluri and van Ryzin (2004a). The authors characterize the optimal policy in terms of "efficient sets" of product offerings, where customers have some probability of purchasing different products from the offered set based on all products in that set. In van Ryzin and Liu (2004), the choice-based revenue management model is extended from the single leg case to consider product offerings for a network. Zhang and Cooper (2005) examine booking limit policies when customers have a choice of flights between the same origindestination pair within a short time period. In a related paper, Cooper et al. (in press) reinforce the necessity of these choice-based extensions, showing how the allocation rules that omit consumer choice can consistently produce poor revenue outcomes. In particular, they study the spiral-down effect - 
where estimates of high-fare demand fall systematically over time because of erroneous modeling assumptions - for Littlewood's rule.

Our model also incorporates consumer choice behavior, but in a way that is somewhat distinct from the earlier works. First, we model choice at an aggregate level, rather than at the individual customer level. Next, we are not considering a strict monopolist environment, in which a single firm controls the product offerings in the market. Rather, we consider a duopoly environment in which two firms offer a similar origin-destination product, but with potential differences in price and other nonprice factors. This is an important departure from the previous choice-based revenue management models, since here the discount pricing firm only has partial control over the menu of products available to customers. Finally, we make the additional assumption that, from the discount firm's perspective, a price only choice model at the aggregate level adequately describes consumer behavior in this environment. That is, a demand capture model based only on price factors is assumed to be sufficient.

Because we consider a duopoly environment, although not in a fully game theoretic way, our work also has some relatedness to the research on competitive inventory models. Interestingly, this stream includes very little research devoted expressly to revenue management. Extensions to the classical newsvendor inventory problem are examined in Lippman and McCardle (1997) and Mahajan and van Ryzin (2001). Belobaba and Wilson (1996) provide an early simulation based study of the impact of revenue management practice on competition in the airline industry. And in their recent work, Netessine and Shumsky (2005) provide the most direct modeling and analysis of competitive revenue management that we know of. We note that each of these papers consider forms of quantity competition, and not price competition that would relate more strongly to our work. As stated above, although we consider a multi-firm environment, our model is not truly game theoretic. The primary advantage of our approach is that we are not forced to make the unnecessary informational assumptions that are required of many of the competitive models.

We develop a general revenue management model for discount providers selling a single product on a single leg, in which a low-cost firm captures exogenous demand by underpricing a fullservice firm. Two specific model formulations are considered: a deterministic price and demand continuous-time version, and a stochastic price and demand discrete-time version. Structural results are derived for the deterministic case, providing insight into the general form of optimal underpricing policies. A more realistic and practically usable model, incorporating stochastic and time-dependent price and demand functions, is studied using an easily implementable Monte Carlo dynamic program 
routine. The stochastic results support the structural insight from the deterministic solution, and illuminate the effect of uncertainty on the underpricing policies.

The remainder of the paper is presented as follows. Section 2 describes in greater detail the lowcost environment that we will consider. The general underpricing model is presented in Section 3 . The deterministic formulation and optimal policy is presented in Section 4, and the stochastic case is presented in Section 5. Pricing examples are presented for both formulations to illustrate the underpricing policies. Section 6 concludes.

\section{Low-Cost Carriers}

We consider the revenue management environment for a low-cost carrier airline such as Westjet in Canada, Southwest in the US or RyanAir in the UK. These firms operate primarily in the shorthaul market, offering point-to-point service, and have limited ability to accommodate connecting traffic. The typical cost structure for these firms is such that they can offer relatively inexpensive fares, but the service level is diminished compared with the full-service carrier. Furthermore, these firms often offer only a single fare class option, and this is the case that we will consider. Then the low-cost carrier (LCC) competes for passenger demand by undercutting the full-service carrier (FSC) on a pricing basis. Conversely, the FSC competes based on connecting traffic, long-haul flights, and many fare class options (offering flexibility in cancelation or schedule changes). The FSC must maximize revenue over a network, has a different cost-structure, and cannot play the same pricing game (or chooses not to cannibalize higher yielding traffic). For this reason, we assume that the FSC will not change their pricing strategy in the presence of an established LCC. Alternately, the LCC may be a subsidiary of the FSC (as is the Jazz airline with respect to Air Canada). In either case, we assume that FSC follows a pricing strategy with no competitive reaction to the LCC's pricing strategy (once it has been established in the market), other than offering some capacity (early on) at reduced prices.

Fig. 2.1 displays a sample price and booking profile for a major US low-cost carrier. The figure displays data for two markets. The solid line is the lowest available fare from a major airline (e.g. American Airlines, United, Delta etc. ..). Bookings for the LCC are the solid series, and the corresponding LCC prices as the unshaded series. The figure indicates that the LCC tends to offer considerable discounts from the FSC price, and that the bookings may indeed be linked to the price gap between the two. 

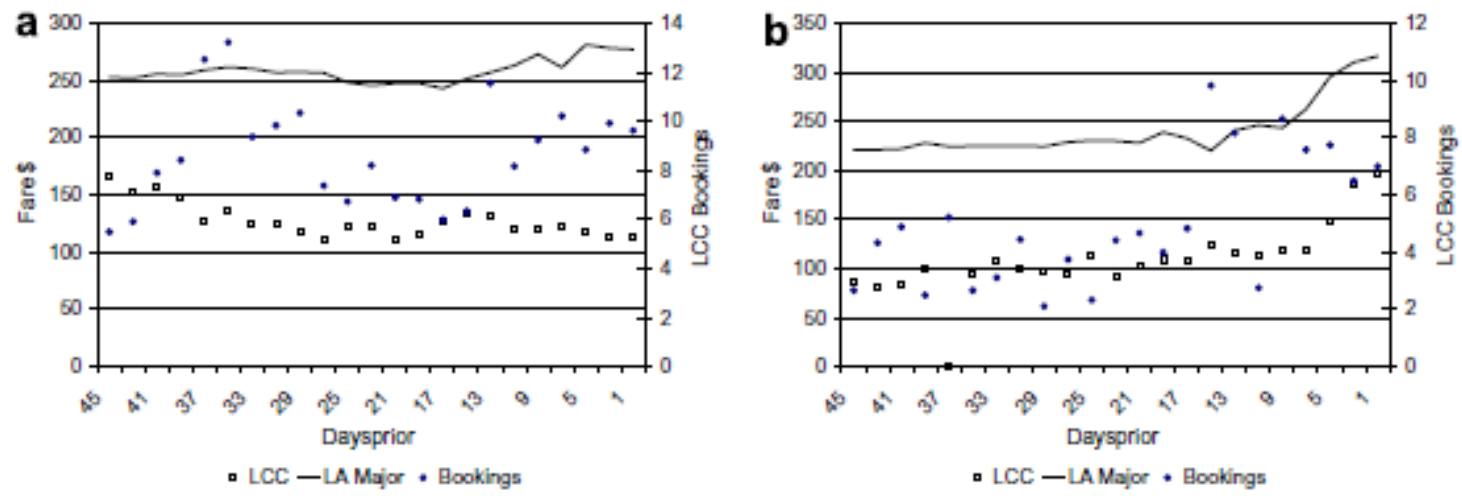

Fig. 2.1. US major LCC prices, bookings and lowest available FSC prices: (a) Route 1; (b) Route 2.

\section{An Underpricing Model}

We now propose a model that captures the dynamics of interest - the revenue implications of a discount provider's underpricing strategy. Let the $\mathrm{LCC}$ apply underpricing control $\mathrm{u}(\mathrm{t})$ at time $\mathrm{t}, \mathrm{t} \in[0, \mathrm{~T}]$ with $\mathrm{T}$ being the time of expiry. It is then natural to suppose that, at any given time, the share of demand captured by the LCC is an increasing function of $u(t)=0$. Several hypothetical demand capture curves are shown in Fig. 3.1, with underpricing control bounded between 0 and 1. For these stylized functions some minimum demand is captured if the LCC matches the FSC price $(u(t)=0)$, and some maximum demand capture is attained if the LCC price falls low enough $(u(t) \rightarrow 1)$.

We assume an exogenous demand process, where customers arrive and request tickets despite the posted prices in the market. While this implies that customers do not act strategically in the timing of their bookings, we do allow that they are still both price and product sensitive at the time of their request. Then we ask, at the time of arrival, and for the FSC and LCC prices at that time, from whom will these price/product-sensitive customers purchase? We assume that, all else equal, customers have some affinity towards the FSC. This could be for many reasons: the ability to have connecting flights, the security of flying with a large airline, a favored frequent-flyer points program, the ability to upgrade tickets, etc. As a result, since the LCC is unable to offer these product enhancements, their ability to capture demand rests entirely on their underpricing strategy. Then the LCC's revenue management objective is to find the underpricing control over the booking horizon to capture demand in a way that maximizes revenue.

We consider a LCC selling inventory in a single fare class for a single leg. The number of seats remaining at time $t$ is $n(t)$, with initial capacity $n(0)=n_{0}$. The salvage value at $T$ of $n(T)$ is zero. The LCC 
underprices the FSC and captures a fraction of the instantaneous demand rate according to the underpricing control $\mathrm{u}(\mathrm{t})$, where $0<\mathrm{u}(\mathrm{t})<1$.



Fig. 3.1. Hypothetical LCC demand capture functions $f(u)$.

The FSC price and the exogenous demand rate follow known differential equations, $\mathrm{P}(\mathrm{t})$ and $\dot{\gamma}(t)$, with $\mathrm{P}(0)=\mathrm{P}_{0}$ and $\mathrm{y}(0)=\mathrm{y}_{0}$. The LCC price $\mathrm{p}(\mathrm{t})$ is derived from the FSC price $\mathrm{P}(\mathrm{t})$ and an underpricing function $f(u)$ as

$$
p(t)=P(t) f(u)
$$

where $0<f(u)<\alpha$. Setting $\alpha<1$ restricts the LCC to never price above the competitor, though this is not necessary in general. The LCC captures a portion of the demand rate through a known demand capture function $g(u)$. The realized demand rate is

$$
d(t)=\gamma(t) g(u)
$$

where $0<\mathrm{g}(\mathrm{u})<1$. The sales rate, the rate of inventory depletion, follows directly from (3.2) as the negative of the captured demand rate 


$$
\frac{\mathrm{d} n}{\mathrm{~d} t}=\dot{n}(t)=-d(t),
$$

with $n(0)=n_{0}$ and restriction $n_{0}<n(t)>\beta$. We note that overbooking can be included in this model by setting $\beta<0$, which would allow inventory to become negative. The resulting instantaneous revenue rate is then

$$
r(t, u)=p(t) d(t)=P(t) \gamma(t) f(u) g(u) .
$$

Finally, we have the usual objective of maximizing total revenue over the booking horizon

$$
\max _{u(t)} \int_{0}^{T} r(t) \mathrm{d} t .
$$

\section{A Deterministic Formulation}

We now specify a deterministic model consistent with the above formulation. The FSC price $P(t)$ changes according to a known constant growth factor $g_{p}$, and the exogenous demand rate changes according to a known constant growth factor $\mathrm{g}_{\mathrm{y}}$, so that $P(t)=\dot{g}_{p} P(t)$ and $\dot{\gamma}(t)=g_{y} y(t)$. The underpricing function is $f(u)=1-u$, and the demand capture function is $g(u)=u$, so that $p(t)=P(t)(1-u)$ and $\mathrm{d}(\mathrm{t})=\mathrm{y}(\mathrm{t}) \mathrm{u}$. In this case $\alpha=1$ since $0<\mathrm{u}<1$ by definition, and so here the control $\mathrm{u}$ is the percentage underpricing of the LCC with respect to the FSC. Though not a requirement of the general model in Section 3, with this formulation we are assuming that the LCC must underprice to capture demand. For simplicity, we will not allow overbooking, so we set $\beta=0$, implying the constraint $n(t)>0$. With this specification, we have the following revenue maximizing optimal control problem:

$$
\max _{u} \int_{0}^{T} P \gamma\left(u-u^{2}\right) \mathrm{d} t
$$

subject to 


$$
\begin{aligned}
& \dot{P}=g_{P} P, \quad P(0)=P_{0}, \\
& \dot{\gamma}=g_{\gamma} \gamma, \quad \gamma(0)=\gamma_{0}, \\
& \dot{n}=-\gamma u, \quad n(0)=n_{0},
\end{aligned}
$$

and

$$
0 \leqslant u \leqslant 1, \quad n \geqslant 0 .
$$

\section{Optimal Policy}

The optimal policy for the model in Section 4 has a analytical solution that takes the general form of bang- bang control. We outline the important features of this policy here; the derivation can be found in Appendix 1.

Define maximal demand capture $d_{\max }$ as the demand that would be captured by maximizing the revenue rate over the entire booking period if there were no inventory constraint:

$$
d_{\max }=\int_{0}^{T} \frac{1}{2} \gamma_{0} \mathrm{e}^{g_{7} t} \mathrm{~d} t .
$$

If $d_{\max }<n_{0}$ the underpricing is trivial. In this case the LCC will underprice at the revenue-maximizing rate over the entire booking horizon since it is not possible to stock-out before time $T$ by doing so. When $d_{\max }$ $>n_{0}$, the LCC must be more strategic in its underpricing. The following three cases assume this to be the case.

When the FSC price is constant $\left(g_{p}=0\right)$, the LCC will maximize total revenue by underpricing over $t \in[0, T]$ to stock-out exactly at time $T$. The optimal policy in this case is

$$
u^{*}(t)=\frac{1}{2}-\frac{C(T)}{2 P},
$$

\section{Where}


(4.2)

$$
C(T)= \begin{cases}P_{0}\left[1-\frac{2 m_{0}}{\gamma_{0} T}\right], & g_{\gamma}=0, \\ P_{0}\left[1-\frac{2 n_{0} g_{7}}{\gamma_{0}\left(e^{57^{7}}-1\right)}\right], & g_{\gamma} \neq 0 .\end{cases}
$$

When $P(t)$ is increasing $\left(g_{P}>0\right)$, the LCC waits until time $\tau_{1}$ to begin underpricing when some minimum price $P\left(\tau_{1}\right)$ is reached. The optimal policy is

$$
u^{*}(t)= \begin{cases}0, & t \in\left[0, \tau_{1}\right), \\ \frac{1}{2}-\frac{C\left(\tau_{1}\right)}{2 P}, & t \in\left[\tau_{1}, T\right],\end{cases}
$$

where

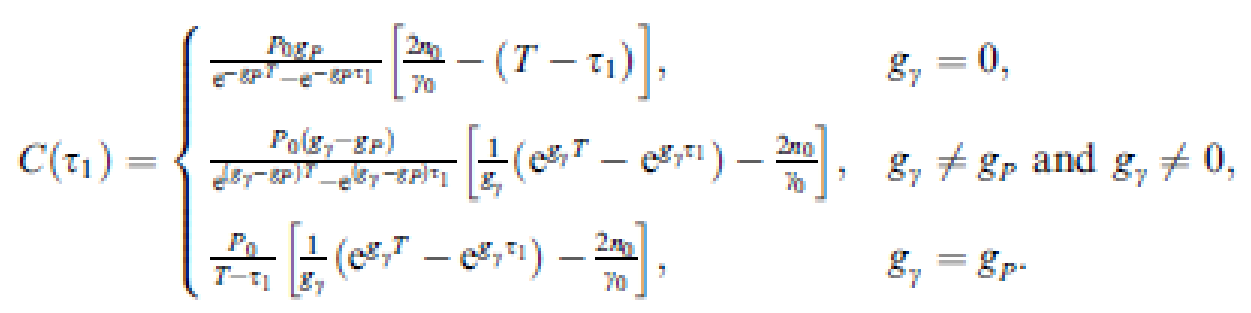

The time $\tau_{1}$ satisfies $P\left(\tau_{1}\right)=C\left(\tau_{1}\right)$ when $\tau_{1}>0$, and can be solved for numerically.

When $P(t)$ is decreasing $\left(g_{P}<0\right)$, the LCC will stop underpricing at time $\tau_{2}$ when some minimum price $\mathrm{P}\left(\tau_{2}\right)$ is reached (stock-out at time $\tau_{2}$ ). The optimal policy is

$$
u^{*}(t)= \begin{cases}\frac{1}{2}-\frac{C\left(v_{2}\right)}{2 P}, & t \in\left[0, \tau_{2}\right], \\ 0, & t \in\left(\tau_{2}, T\right],\end{cases}
$$

where 
(4.8)

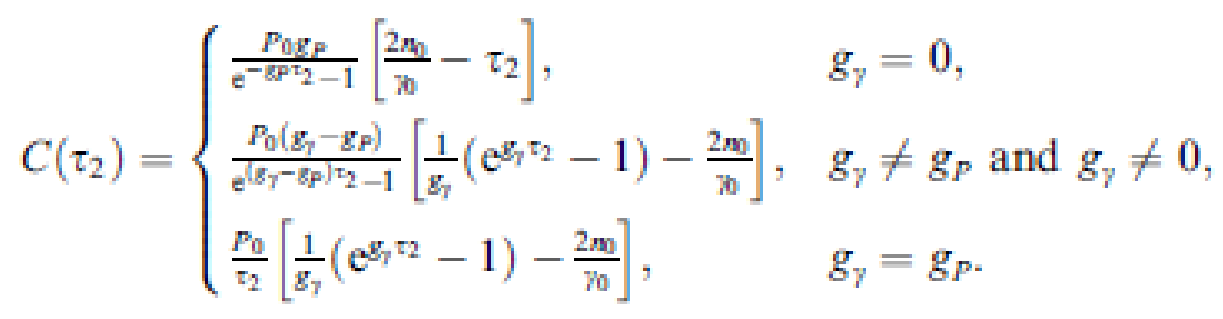

The time $\tau_{2}$ satisfies $P\left(\tau_{2}\right)=C\left(\tau_{2}\right)$ when $\tau_{2}<T$, and can be solved for numerically.

\section{Deterministic Pricing Examples}

The following examples illustrate the optimal control for three different price and demand scenarios. The first two are typical of airlines, with a gradual increase in demand and price as departure approaches. The third is more typical of a fashion item, where prices and demand tend to decrease with time.

The first example, shown in Fig. 4.1, displays an increasing FSC price and the optimal underpricing path for the LCC in panel (a), with panel (b) displaying capacity $(n(t))$ and the control $(u(t))$. In this case the LCC underprices over the entire booking period, and runs out of capacity just prior to departure.

The second example, shown in Fig. 4.2, also has increasing FSC price. But in this case, demand is higher, and so the LCC delays underpricing for some time. Eventually the LCC begins to underprice, and does so over the remaining horizon, and as in the first example, underprices in order to run out of capacity just prior to departure. 
a

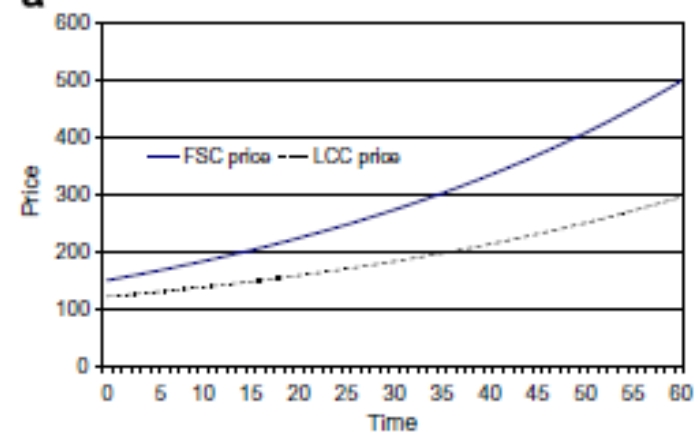

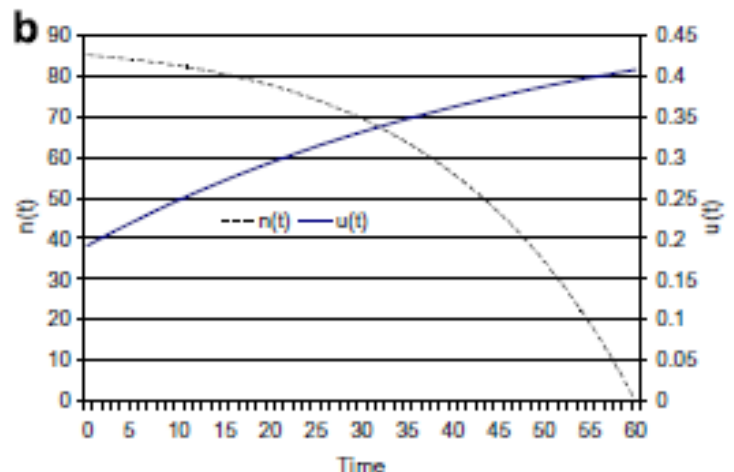

Fig. 4.1. Example 1 - increasing prices. (a) Prices (b) Control and Capacity.


Fig. 4.2. Example 2 - Increasing prices and high demand: (a) prices; (b) control and capacity.
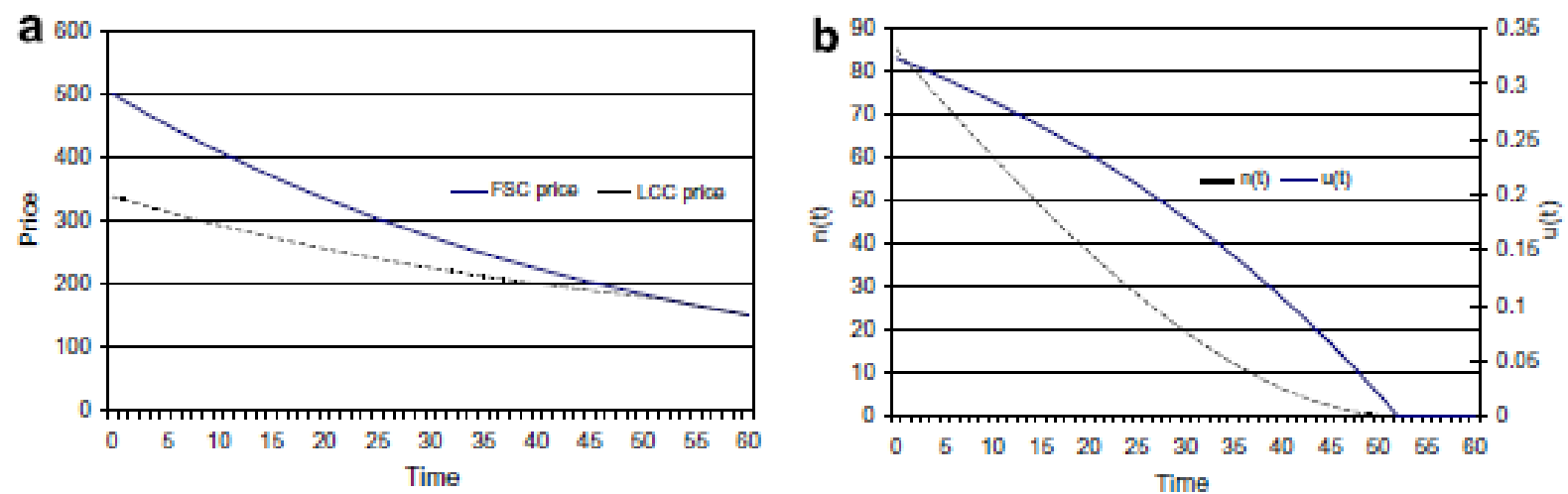

Fig. 4.3. Example 3 - Decreasing prices. (a) prices; (b) control and capacity.

In the final example, Fig. 4.3, prices are decreasing. In contrast to the first two examples, here the firm underprices aggressively early in the booking period to capture as much high value demand as possible, and as a result stocks-out early.

In each of these cases, the optimal policy is a form of bang-bang control. The LCC firm either (1) underprices over the entire horizon, (2) waits until some switching time before underpricing over the 
remaining horizon, or (3) underprices until some switching time, after which underpricing is not resumed. While the applicability of this control for practical purposes is limited, due in part to the rigid nature of the deterministic model, these results do provide some first-order insight into a discount providers optimal pricing strategy. In general, these results suggest that, depending on the product and industry, discount pricing firms should adopt either an underprice-early, underprice-late, or a periodlong underpricing strategy in order to maximize revenue. We now explore how these results translate to the case of uncertain price and demand.

\section{Discount Pricing Under Uncertainty}

The preceding analysis has provided a foundation for understanding the optimal underpricing behavior for a discount service provider. As noted above however, the uncertain nature of demand presents the greatest barrier to effective revenue management. This may be especially true in the case of the discount service provider, since in reality both the FSC price and the demand rate are stochastic and unpredictable. Furthermore, demand (and sales) quantities for most revenue managing firms are not continuous, as modeled above, but discrete - typically integer valued. In this section we extend the model and specification from Sections 3 and 4 to better account for these realities. Specifically, the previously deterministic model is altered to allow the FSC price $\mathrm{P}(\mathrm{t})$ and the demand rate $\mathrm{c}(\mathrm{t})$ to follow stochastic processes, and to require the sales amounts to be integer valued. A Monte Carlo dynamic programming routine is proposed for identifying the optimal underpricing control in this stochastic setting. Discrete-time, rather than continuous-time as above, is used to accommodate the proposed methodology.

\section{A Stochastic Formulation}

The deterministic model of Section 4 has provided a qualitative understanding of the optimal underpricing control for a discount service provider. To move beyond this qualitative insight, and to a more practically usable model, several extensions are needed. Here, we propose an alternate discretetime, discrete-capture, stochastic- price and -demand model.

Let the time increment be $\Delta \mathrm{t}=1$, so that $\mathrm{t} \in 2[0 ; 1 ; 2 ; \ldots ; \mathrm{T}]$. For notational convenience, in this section the dependence on time $t$ is denoted by a subscript on the variables whenever the meaning will be clear. Let the FSC price follow the discrete-time stochastic process 


$$
\Delta P_{t}=g_{P}(t) P_{t}+\sigma_{P}(t) P_{t} \varepsilon
$$

where $g_{P}(t)$ and $\sigma_{P}(t)$ are the drift and volatility of the FSC price, respectively, and $\varepsilon$ is a standard normal random variable. Similarly, let the exogenous demand rate follow

$$
\Delta \gamma_{t}=g_{\gamma}(t) \gamma_{t}+\sigma_{\gamma}(t) \gamma_{t} \varepsilon
$$

where $g_{y}(t)$ and $\sigma_{p}(t)$ are the drift and volatility of demand, respectively. Eqs. (5.1) and (5.2) take the well known form of geometric Brownian Motion with (time-dependent) drift (Hull, 2003). These are quite natural and flexible stochastic extensions of their deterministic counterparts above. For simplicity and ease of comparison, we retain the underpricing and demand capture functions from Section 4, namely $f(u)=1-u$ and $g(u)=u$.

With this alternate specification, we seek to maximize expected revenue over the bookinghorizon:

$$
\max _{*} E\left[\sum_{t=0}^{T} P_{t} \gamma_{l}\left(u_{t}-u_{t}^{2}\right)\right]
$$

subject to Eqs. (5.1) and (5.2), and

$$
\Delta n_{l}=-\gamma_{4} u_{i},
$$

and

$$
0 \leqslant u \leqslant 1, \quad n \geqslant 0 \text {, }
$$

and with underpricing control additionally restricted to the set $u_{i} \in\left[0, \frac{1}{\gamma_{l}}, \frac{2}{\gamma_{l}}, \ldots\right]$ so that $\Delta n_{t} \in$ $[0,1,2, \ldots]$

\section{A Monte Carlo Dynamic Programming Solution}

In a stochastic environment we seek to find the optimal underpricing for the present time period, $t$, for which $\mathrm{P}_{t}$ and $\mathrm{y}_{\mathrm{t}}$ are known. A Monte Carlo dynamic programming routine, which is quite 
easy to implement, can be used to identify this control. The basic approach is as follows. Simulate a price and demand path realization for the entire horizon. For this realization, we can identify the optimal underpricing via a dynamic program. Finally, simulate and solve $\mathrm{N}=$ many times, in Monte Carlo fashion, and aggregate the results to find the optimal control. We now provide the specifics of this approach in more detail.

Suppose that at time $t=0$, the current time, we know how the FSC price and the demand rate will evolve for the entire horizon, i.e. $P_{t}$ and $y_{t}$ are known $\forall \in[0, \ldots, T]$. Then we can solve the dynamic program

$$
V_{t}\left(n_{t}, P_{t}, \gamma_{t}\right)=\max _{t_{t}}\left[P_{t} \gamma_{t}\left(u_{t}-u_{t}^{2}\right)+V_{t+1}\left(n_{t}-\Delta n_{t}, P_{t+1}, \gamma_{t+1}\right)\right],
$$

with final condition

$$
V_{T}\left(n_{T}, P_{T}, \gamma_{T}\right)=\max _{\alpha_{T}} P_{T} \gamma_{T}\left(u_{T}-u_{T}^{2}\right)
$$

for the optimal underpricing control $u_{0}^{*}$ over the inventory state-space $n_{t} \in\left[0, \ldots, n_{0}\right]$. This provides the optimal time $t=0$ control for a given path. Since we do not know the actual path, we must simulate a large number of path realizations to find the control that works best on average. Let there be $\mathrm{N}$ such path realizations, indexed by $\mathrm{i}=1 ; \ldots$; . Then the price and demand realizations for the ith iteration follow from Eqs. (5.1) and (5.2):

$$
P_{t}^{j}=P_{t=1}^{j} e^{g_{r}(t)+\sigma_{r}(t) \mathrm{s}},
$$

and

$$
y_{t}^{i}=\gamma_{t=1}^{t} \mathrm{e}^{\varepsilon_{r}(t)+\sigma_{y}(t) \mathrm{s}},
$$

where $P_{0}^{i}=P_{0}$ and $\gamma_{0}^{i}=\gamma_{0}$ are known, and e is a draw from a standard normal random variable. The preferred underpricing control $u_{0}^{*}$, the one that works best on average, is simply 


$$
u_{0}^{*}=\frac{1}{N} \sum_{i=1}^{N} u_{0}^{* i} \text {. }
$$

\section{Stochastic Pricing Examples}

Before exploring the impact that randomness in price and demand have on the underpricing policies, it is instructive to compare the discrete capture pricing, with demand and price volatilities set to zero, with the policy from the deterministic/continuous model from Section 4. Fig. 5.1 shows the optimal prices for both models in panel (a), and the associated optimal control and inventory levels in panel (b). We observe immediately that the discrete-capture model has much less smooth price and control then the continuous-capture model. We also see that the underpricing begins later, and has a piecewise-linear nature - a direct result of requiring integer demand capture. Interestingly, in panel (b) we see that despite the relatively large fluctuations in discrete-capture underpricing control, the resulting inventory levels for the two controls track quite closely. Fig. 5.2 shows the demand capture associated with the discrete capture control from this example. Comparing this illustration with Fig. 5.1 shows clearly that the price-jump discontinuities occur when the optimal price control shifts to a different integer value of demand capture.

We now present several examples to show the effect that randomness in FSC price and exogenous demand have on the optimal underpricing behavior. We consider three cases: (1) volatility in price only, (2) volatility in demand only, and (3) volatility in both demand and price. In each case, for comparison, we show the optimal underpricing over the horizon as though FSC price and exogenous demand evolve according to their expectations.

Fig. 5.3 displays the underpricing under FSC price uncertainty. Three LCC price series are shown: no FSC price volatility (p_0), low volatility in FSC price (p_low), and high volatility in FSC price (p_high). When there is no uncertainty in FSC price, the underpricing is as before, increasing gradually over the horizon. As volatility in FSC price is increased, a smoothing of the LCC price can be seen. That is, the LCC increases underpricing earlier in the booking period, and decreases underpricing later in the booking period, so that a more constant underpricing is observed over the horizon. Fig. 5.4 (demand volatility) and Fig. 5.5 (demand and price volatility) display similar behavior for low and high volatility cases. 



Fig. 5.1. Comparison of continuous and discrete demand capture prices, inventory and control: (a) prices; (b) inventory and control.

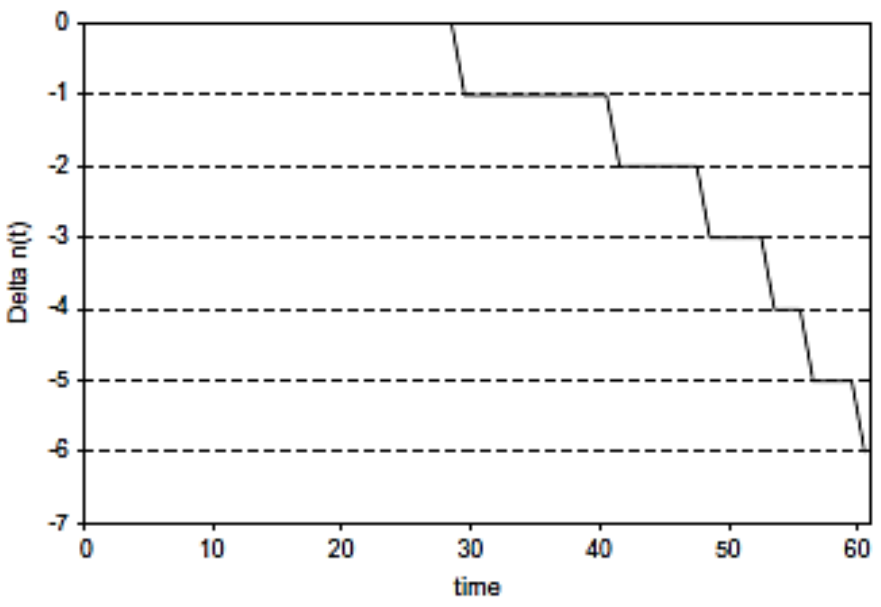

Fig. 5.2. $\Delta n_{t}$ - discrete demand capture.

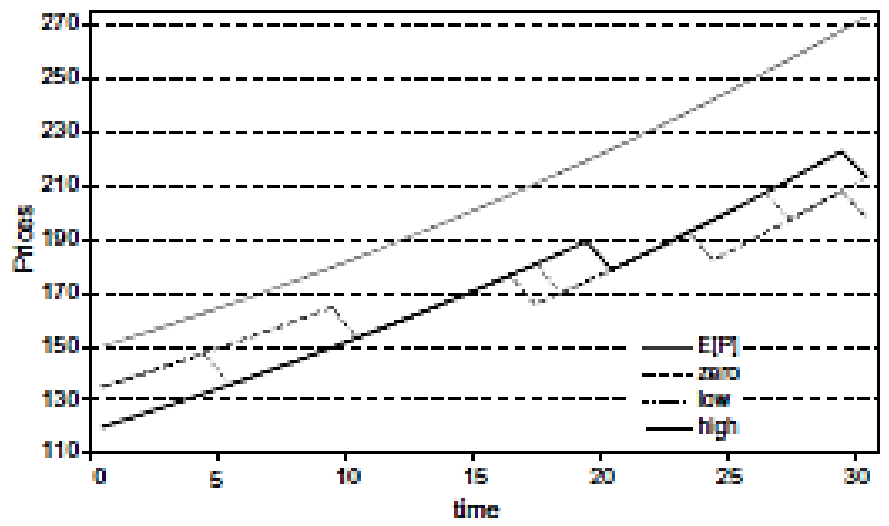

Fig. 5.3. Underpricing with uncertainty in FSC price. 




Fig. 5.4. Underpricing with uncertainty in exogenous demand.

In all cases, there is a similar effect of uncertainty on the underpricing policies. In general, as volatility in any of the variables increases, the firm applies a more conservative and consistent underpricing strategy. This is an intuitively appealing result: when the firm has more uncertainty about the future evolution of market demand and price, they attempt to smooth their revenue and demand capture streams by applying a more uniform underpricing control over the booking period. This observation in no way implies that the LCC should not dynamically price. Rather, it suggests that, given a highly stochastic market, their dynamic pricing should track quite closely with that of the FSC - taking it's same basic shape - by applying a relatively constant underpricing control. Furthermore, in less stochastic markets, the firm should apply the more aggressive strategies of early or late underpricing as the deterministic controls suggest.

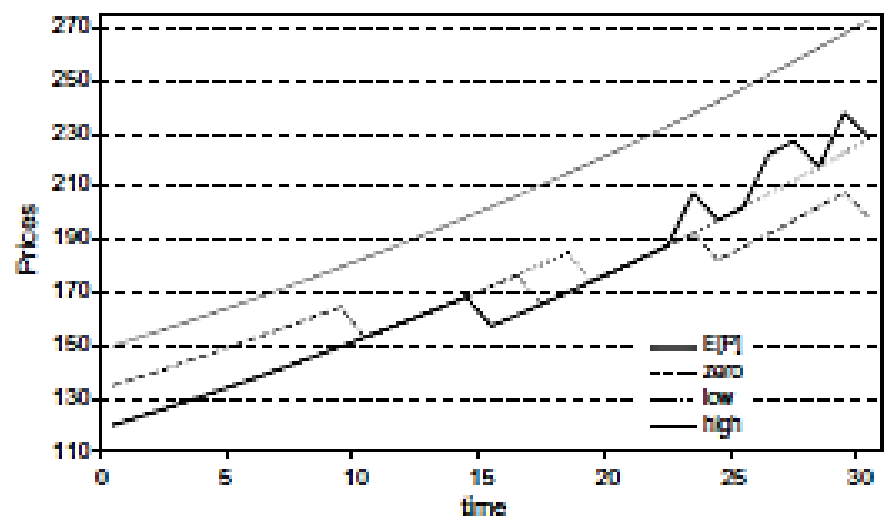

Fig. 5.5. Underpricing with uncertainty in both FSC price and demand. 


\section{Conclusions}

We have developed revenue management models for discount service providers. Structural results have been obtained for a deterministic formulation of this model, which define the general form of an optimal underpricing policy. These results have been extended to a more realistic stochastic environment, and the effect of uncertainty has been examined.

These results depend on several assumptions that could be relaxed in future work. In particular, extending this work to allow the FSC to react competitively may provide additional valuable insight. Recently, several full-service carriers have also been experimenting with more transparent fare structures - offering one-way service with fewer restrictions - providing the potential for competing more directly on price. An analysis of these structural changes, and their impact on a LCC's pricing strategy would provide a nice complement to the present work.

\section{Appendix 1. Optimal policy derivation}

In this section we restate the control problem from Section 4 and provide its full solution. We have the revenue maximizing control problem:

$$
\max _{u} \int_{0}^{T} P \gamma\left(u-u^{2}\right) \mathrm{d} t
$$

subject to

(A1)

$$
\dot{P}=g_{P} P, \quad P(0)=P_{0},
$$

(A2)

$$
\dot{\gamma}=g_{\gamma} \gamma, \quad \gamma(0)=\gamma_{0},
$$

(A3)

$$
\dot{n}=-\gamma u, \quad n(0)=n_{0} .
$$

and

(A4)

$0 \leqslant u \leqslant 1, \quad n \geqslant 0$. 
The Hamiltonian and Lagrangian are

$$
H=P \gamma\left(u-u^{2}\right)+\lambda_{p} g_{P} P+\lambda_{\gamma} g_{\gamma} \gamma-\lambda_{k} \gamma u
$$

and

$$
L=H+\mu_{1} u+\mu_{2}(1-u)+v n,
$$

respectively, where $I 1,12$ and $m$ are the multipliers for the constraints in (A4). The necessary conditions for optimality are as follows. The Lagrangian is maximized with respect to the control $u$, giving

(A5)

$$
\frac{\partial L}{\partial u}=0=P \gamma(1-2 u)-\lambda_{n} \gamma+\mu_{1}-\mu_{2}
$$

The adjoint equations for the three state variables are

(A6)

$$
\begin{aligned}
& \dot{\lambda}_{P}=-\frac{\partial L}{\partial P}=-\lambda_{P} g_{P}-\gamma\left(u-u^{2}\right), \\
& \dot{\lambda}_{\gamma}=-\frac{\partial L}{\partial y}=\lambda_{n} u-\lambda_{\gamma} g_{\gamma}-P\left(u-u^{2}\right), \\
& \dot{\lambda}_{n}=-\frac{\partial L}{\partial n}=-v,
\end{aligned}
$$

with time $T$ transversality conditions $\lambda_{P}(T)=\lambda_{Y}(T)=0$, and $\lambda_{n}(T)=\alpha$ with $\alpha n(T)=0$ (see Hartl et al., 1995). The complementary slackness conditions for the constraints in (A4) are

(A7)

$\mu_{1} \geqslant 0, \quad \mu_{1} u=0$,

(A8)

$\mu_{2} \geqslant 0, \quad \mu_{2}(1-u)=0$,

(A9)

$v \geqslant 0, \quad v n=0$.

A possible discontinuity in $\lambda_{n}$ at a contact time $\tau$ of the constraint $n>0$ is provided by the jump condition 
(A10)

$$
\begin{aligned}
& \lambda_{n}\left(\tau^{-}\right)=\lambda_{n}\left(\tau^{+}\right)+\eta(\tau), \\
& H^{*}\left[\tau^{-}\right]=H^{*}\left[\tau^{+}\right]-\eta(\tau),
\end{aligned}
$$

with $\eta(\tau)>0$, and $\eta(\tau) n[t]=0$. Here, $\tau^{+}$and $\tau^{-}$indicate right and left-hand limits respectively, while $[\cdot]$ indicates that notation for additional dependencies has been suppressed.

From Eqs. (A5), (A6), and (A9) we have that,

$$
u= \begin{cases}\frac{1}{2}-\frac{\lambda_{n}-\mu_{1}+\mu_{2}}{2 P_{\gamma}}, & n(t) \geqslant 0, \\ 0, & n=0 .\end{cases}
$$

From the state equations and initial conditions in (A1) and (A2), it follows that

$$
P(t)=P_{0 e^{\varepsilon s t}}
$$

and

$$
\gamma(t)=\gamma_{0} \mathrm{e}^{s \gamma^{t}}
$$

so that competitor's price and exogenous demand are both monotonic. The instantaneous revenue rate $r(t)$ is then

$$
r(t)=P(t) \gamma(t)\left(u-u^{2}\right)=P_{0} \gamma_{0} \mathrm{e}^{\left(g r+g_{\gamma}\right) t}\left(u-u^{2}\right) .
$$

For any time $\tau, r(\tau)$ is concave in $u$ with its maximum at $u=1 / 2$, while the rate of demand capture, the sales rate, is linearly increasing in control. It is therefore never optimal to underprice by more than a half, and

$$
0 \leqslant u^{*}(t) \leqslant \frac{1}{2} \text {. }
$$

We can now obtain some simplification for the general form of control. First, it follows from (A12) and (A8)that $\mu_{2}=0$, since the constraint $u<1$ will never be tight. Second, from (A7), whenever $u \neq 0$ it must be true that $\mu_{1}=0$. Finally, from (A6) and (A9) we obtain

$$
\lambda_{n}= \begin{cases}C, & n>0 \\ 0, & n=0\end{cases}
$$


where $\mathrm{C}$ is a constant. The general form of control in Eq. (A11) can now be rewritten as

(A13)

$$
u= \begin{cases}\frac{1}{2}-\frac{c}{2 P}, & n(t)>0, \\ 0, & n(t)=0 .\end{cases}
$$

To complete the solution, we need to find when to apply this control, and what is the value of $C$.

Define maximal demand capture $d_{\max }$ as the demand that would be captured by maximizing the revenue rate over the entire booking period with no consideration of the inventory constraint:

$$
d_{\operatorname{mas}}=\int_{0}^{T} \frac{1}{2} \% e^{2} \mathrm{~d} t
$$

If $\mathrm{d}_{\max }<\mathrm{n}_{0}$ the underpricing is trivial. In this case the LCC will underprice at the revenue-maximizing rate over the entire booking horizon since it is not possible to stock-out before time $\mathrm{T}$ by doing so.

When $d_{\max }>n_{0}$, the LCC must be more strategic in its underpricing. The LCC underprices to capture a portion of the exogenous revenue rate $R(t)=P(t) y(t)=P_{0} y_{0} e^{(g r+g y) t}$, which is monotonic in $t$. Recall that competitor's price $\mathrm{P}(\mathrm{t})$ and the exogenous demand $y(\mathrm{t})$ are also both monotonic in $\mathrm{t}$. These monotonicity results lead to three cases to be examined: $(1) P(t)$ is constant $\left(g_{P}=0\right) ;(2) P(t)$ is monotonically increasing $\left(g_{P}>0\right)$; and $(3) P(t)$ is monotonically decreasing $\left(g_{P}<0\right)$. We now address each of these cases separately.

When competitor's price is constant $\left(g_{P}=0\right)$, the LCC will maximize total revenue by underpricing to stock-out exactly at time T. Solving

$$
\int_{0}^{T} \gamma u \mathrm{~d} t=\int_{0}^{T} \gamma\left(\frac{1}{2}-\frac{C}{2 P}\right) \mathrm{d} t=n_{0}
$$

for $C$ yields Eq. (4.4).

When $P(t)$ is monotonically increasing $\left(g_{P}>0\right)$, it will not be optimal to begin underpricing until time $s 1$ when some minimum price $P\left(\tau_{1}\right)$ is reached. In this case, the maximum total revenue will be attained by underpricing to stock-out at time $T$ by underpricing over the interval $t \in\left[\tau_{1} ; T\right]$. Solving

$$
\int_{0}^{T} \gamma u \mathrm{~d} t=\int_{0}^{T} \gamma\left(\frac{1}{2}-\frac{C}{2 P}\right) \mathrm{d} t=n_{0}
$$

for $C$ yields Eq. (4.6). 
When $\mathrm{P}(\mathrm{t})$ is monotonically decreasing $\left(\mathrm{g}_{\mathrm{P}}<0\right)$, it will not be optimal to continue underpricing after time $\tau_{2}$ when some minimum price $P\left(\tau_{2}\right)$ is reached. In this case, the maximum total revenue will be attained by underpricing to stock-out at time $\tau_{2}$ by underpricing over the interval $t \in\left[0, \tau_{2}\right]$. Solving

$$
\int_{0}^{t_{2}} \gamma u \mathrm{~d} t=\int_{0}^{t_{2}} \gamma\left(\frac{1}{2}-\frac{C}{2 P}\right) \mathrm{d} t=n_{0}
$$

for $C$ yields Eq. (4.8).

We can now find the optimal state trajectory $n *(t)$ by solving Eq. (A3) with boundary conditions $\mathrm{n}(0)=\mathrm{n}_{0}$ for $\mathrm{g}_{\mathrm{P}}<0$ and $\mathrm{gP}<0$, and $\mathrm{n}\left(\tau_{1}=\mathrm{n}_{0}\right.$ for $\mathrm{g}_{\mathrm{P}}>0$. For $\mathrm{g}_{\mathrm{P}}=0$ we obtain

$$
n^{*}(t)= \begin{cases}n_{0}+\frac{\hbar_{0}}{2}\left[\frac{c}{P_{0}}-1\right], & g_{\gamma}=0, \\ n_{0}+\frac{\eta_{0}}{2 z_{y}}\left[\frac{1}{2}-\frac{c}{2 P_{0}}\right]\left(1-e^{s} y^{2}\right), & g_{\gamma} \neq 0 .\end{cases}
$$

For $g_{p}>0$ we obtain $n^{*}(t)=n_{0}$ over $t \in\left[0, \tau_{1}\right]$, and

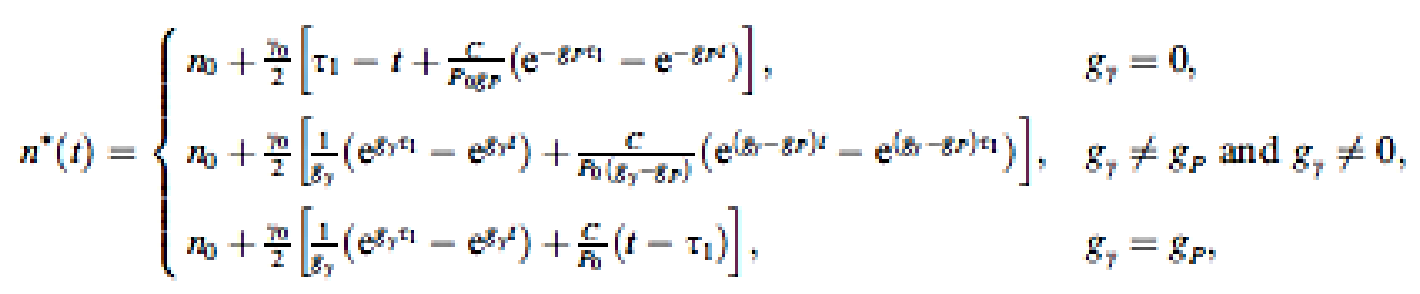

over $\mathrm{t} \in\left[\tau_{1}, \mathrm{~T}\right]$. For $\mathrm{g}_{\mathrm{P}}<0$ we obtain

(A16)

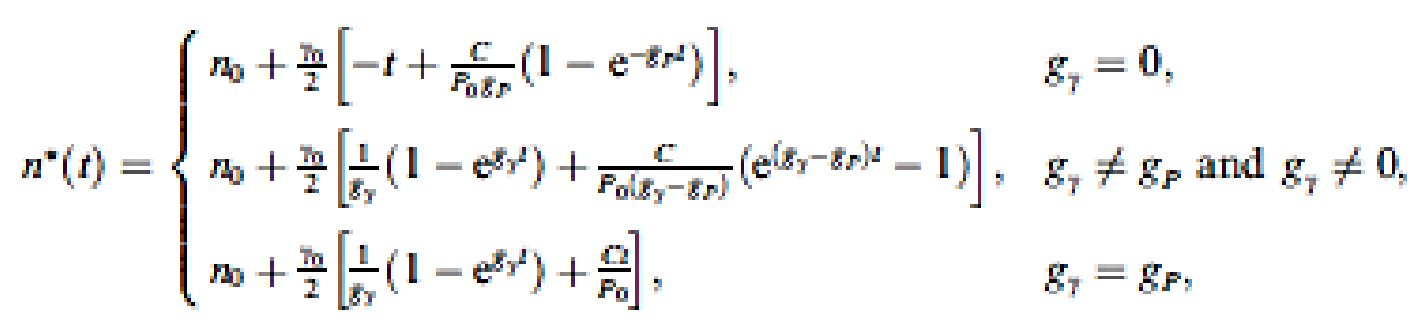

and $n^{*}(t)=0$ over $t \in\left[\tau_{2}, T\right]$. For completeness, we note that there is a jump discontinuity in $\lambda_{n}$ at $\tau_{2}$ when the boundary $n(t)>0$ becomes tight (see (A10)). However, this jump does not affect the solution since no 
seats are sold after this point and we will never exit this boundary. Therefore the value of $\lambda_{n}$ becomes unimportant once the boundary is reached.

\section{References}

Belobaba, P., 1987. Airline yield management: An overview of seat inventory control. Transportation Science 21, 63-73.

Belobaba, P., Wilson, J., 1996. Impacts of yield management in competitive airline markets. Journal of Air Transport Management 3 (1), 3-9.

Bitran, G., Caldentey, R., 2003. An overview of pricing models for revenue management. Manufacturing and Service Operations Management 5, 203-229.

Cooper, W., Homem-de-Mello, T., Kleywegt, A., in press. Models of the spiral-down effect in revenue management.

Elmaghraby, W., Keskinocak, P., 2003. Dynamic pricing in the presence of inventory considerations: Research overview, current practices, and future directions. Management Science 49 (10), 1287-1309.

Feng, Y., Gallego, G., 1995. Optimal starting times for end-of-season sales and optimal stopping times for promotional fares. Management Science 41, 1371-1391.

Feng, Y., Gallego, G., 2000. Perishable asset revenue management with markovian time dependent demand intensities. Management Science 46, 941-956.

Feng, Y., Xiao, B., 2000a. A continuous-time yield management model with multiple prices and reversible price changes. Management Science 46 (5), 644-657.

Feng, Y., Xiao, B., 2000b. Optimal policies of yield management with multiple predetermined prices. Operations Research 48, 332-343, March-April.

Gallego, G., van Ryzin, G., 1994. Optimal dynamic pricing of inventories and stochastic demand over finite horizons. Management Science 40, 999-1020.

Gallego, G., van Ryzin, G., 1997. A multi-product dynamic pricing problem and its applications to network yield management. Operations Research 45, 24-41.

Hartl, R., Sethi, S., Vickson, R., 1995. A survey of the maximum principles for optimal control problems with state constraints. Society for Industrial and Applied Mathematics 37 (2), 181-218.

Hopperstad, C., Belobaba, P., 2004. Algorithms for revenue management in unrestricted fare markets. AGIFORS Reservations and Yield Management Study Group (Auckland, New Zealand). 
Hull, J.C., 2003. Options, Futures and Other Derivatives, fifth ed. Prentice Hall, Upper Saddle River, New Jersey.

Lippman, S., McCardle, K., 1997. The competitive newsboy. Operations Research 45 (1), 54-65.

Littlewood, K., 1972 12. Forecasting and control of passenger bookings. In: AGIFORS Symposium Proceedings (Nathanya, Israel).

Mahajan, S., van Ryzin, G., 2001. Inventory competition under dynamic consumer choice. Operations Research 49 (5), 646-657.

McGill, J., van Ryzin, G., 1999. Revenue management: research overview and prospects. Transportation Science 33 (2), 233-256.

Netessine, S., 2006. Dynamic pricing of inventory/capacity with infrequent price changes. European Journal of Operational Research 174 (1), 553-580.

Netessine, S., Shumsky, R., 2005. Revenue management games: Horizontal and vertical competition. Management Science 51 (5), 813-831.

Pfeifer, P., 1989. The airline discount fare allocation problem. Decision Science 20, 149-157.

Talluri, K., van Ryzin, G., 2004a. Revenue management under a general discrete choice model of consumer behavior. Management Science 50 (1), 15-33.

Talluri, K., van Ryzin, G., 2004b. The theory and practice of revenue management, first ed. Klewer Academic Publishers, Boston, Ma.

Tavana, H., 2004. Optimal airline seat allocation in a fenceless environment using expected revenue maximization. AGIFORS Reservations and Yield Management Study Group (Auckland, New Zealand).

van Ryzin, G., Liu, Q., 2004. On the choice-based linear programming model for network revenue management (August).

Weatherford, L., Bodily, S., 1992. A taxonomy and research overview of perishable-asset revenue management: yield management, overbooking and pricing. Operations Research 40, 831-844.

Wilson, J., Anderson, C., Kim, S.-W., 2006. Optimal booking limits in the presence of strategic consumer behavior. International Transactions in Operational Research 13, 99-110.

You, P., 1999. Dynamic pricing in airline seat management for flights with multiple flight legs. Transportation Science 33, 192-206.

Zhang, D., Cooper, W., 2005. Revenue management for parallel flights with customer-choice behavior. Operations Research 53 (3), 415-431, May-June. 
Zhao, W., Zheng, Y., 2000. Optimal dynamic pricing for perishable assets with nonhomogeneous demand. Management Science 46, 375- 388. 